\title{
EQUIDAD EN SALUD EN LA REGIÓN MÁS DESIGUAL DEL MUNDO: UN RETO DE POLÍTICAS PÚBLICAS EN AMÉRICA LATINA
}

\author{
HEALTH EQUITY IN THE WORLD'S MOST UNEQUAL REGION: A CHALLENGE FOR \\ PUBLIC POLICY IN LATIN AMERICA
}

Patricia Frenz $z^{1,2, a}$, Daniel Titelman ${ }^{2, b}$

Escuela de Salud Pública, Facultad de Medicina, Universidad de Chile. Santiago, Chile.

División de Financiamiento para el Desarrollo, Comisión Económica de América Latina y el Caribe de Naciones Unidas. Santiago, Chile.

Médico, especialista en Salud Pública; ${ }^{b}$ economista, PhD

Recibido: 20-09-2013; Aprobado: 16-10-13

\begin{abstract}
RESUMEN
La redemocratización ha transformado la agenda social y el rol del Estado en América Latina con un compromiso creciente con la equidad y la justicia sanitaria que está tensionado por las profundas desigualdades socioeconómicas. Los esfuerzos por universalizar el derecho a la salud han llevado a desarrollar diversas políticas públicas, cuyo alcance depende del entendimiento de los conceptos de salud y equidad. El foco de acción se ha concentrado en reformas al sistema de salud y solo recientemente hay esbozos de políticas intersectoriales que abordan los determinantes sociales estructurales. Además, si la equidad en salud es el norte la estrategia predominante de establecer garantías mínimas no puede ser la respuesta final, sino un paso en el camino hacia la igualdad. Por último, avanzar hacia la cobertura universal del derecho a la salud requiere fortalecer capacidades institucionales de los gobiernos relacionadas con políticas públicas, con una mirada intersectorial y participativa.
\end{abstract}

Palabras clave: Equidad en salud; Políticas públicas de salud; Reforma de la atención de salud; Accesibilidad a los servicios de salud; América Latina (fuente: DeCS BIREME).

\begin{abstract}
Re-democratization has transformed the social agenda and the role of the state in Latin America with a growing commitment to health equity and social justice, yet these aspirations are strained by the region's profound socioeconomic inequalities. Efforts to provide universal coverage to the right to health have led to the development of a variety of public policies, whose scope depends on how the concepts of health and equity are understood. In general, policy action has centered on health system reforms and only recently on integrated intersectorial action to address wider social determinants of health, particularly structural determinants. Furthermore, if the goal is health equity the predominant minimum standards approach cannot be the final answer, but only a step on the road to equality. Finally, realizing universal coverage of the right to health through public policy requires the strengthening of governmental institutional capacities with an intersectorial and participatory lens.
\end{abstract}

Key words: Equity in health; Health public policy; Health care reform; Health services accessibility; Latin America (source: MeSH NLM).

\section{INTRODUCCIÓN}

Si bien en los últimos veinte años América Latina ha logrado avances en la lucha contra la pobreza, ella es hoy en día la región más desigual del mundo ${ }^{(1,2)}$. Esta desigualdad, que refleja factores estructurales en lo económico y social, también se ha trasladado al ámbito de la salud en sus diversos aspectos. Ya sea en la posibilidad y capacidad de acceder a los servicios de salud, así como en las condiciones de vida y trabajo que afectan la salud de la población, configurando lo que se ha denominado los determinantes sociales de la salud que producen diferencias en el nivel de salud y enfermedad de acuerdo a las condiciones socioeconómicas.

Los derechos sociales han adquirido una creciente importancia en la agenda de políticas públicas de la región como reflejo de los nuevos procesos de democratización. Cada vez más se reconocen las limitaciones del mercado de trabajo para servir de base a sistemas de seguridad social y de salud que cumplan a cabalidad los preceptos de igualdad, universalidad, solidaridad e inclusión. Es por ello que un

Citar como: Frenz P, Titelman D. Equidad en salud en la región más desigual del mundo: un reto de políticas públicas en América Latina. Rev Peru Med Exp Salud Publica. 2013;30(4):665-70. 
elemento central para revertir la fuerza inercial de las desigualdades sociales es mediante el rol decisivo del Estado y las políticas públicas ${ }^{(1)}$.

Es así como, en el ámbito de la equidad en salud, muchos países de la región se han adherido a los principios de las diversas iniciativas globales como la declaración de Alma Ata, la Comisión de Determinantes Sociales de la Salud y la visión amplia de cobertura sanitaria universal. Todos estos movimientos llamaban a la acción decidida de la política pública para alcanzar salud con equidad para toda la población (3).

En lo que sigue de este artículo discutiremos algunos desafíos que los países de América Latina deben superar para transformar la preocupación general por la desigualdad social en salud en acciones concretas de políticas públicas.

\section{LA CONCEPTUALIZACIÓN DE SALUD Y EL ÁMBITO DE LAS POLÍTICAS}

La salud, en cuanto anhelo de igualdad traducible en políticas públicas, tiene sus raíces en la equidad, la universalidad, la solidaridad y la inclusión (4). Comúnmente se entiende por equidad en salud el esfuerzo de reducir diferencias sanitarias entre grupos sociales, que son sistemáticas, evitables y consideradas injustas ${ }^{(5)}$. Las preguntas que surgen de esta definición son, en consecuencia, qué tipos de desigualdades en salud son injustas y, por lo tanto, cuáles deben ser abordadas mediante la política pública.

Las respuestas a estas preguntas condicionan la definición de las políticas y, por lo mismo, el potencial que tienen de alcanzar la equidad en salud ${ }^{(6)}$. Podemos identificar dos aproximaciones que están presentes en las distintas estrategias de políticas de equidad en salud seguidas por los países, representadas en la Figura 1. La primera de ellas se enfoca a tratar o prevenir el daño en la salud, actuando principalmente a través de la atención de la salud pero también la promoción de la salud. Esta última persigue una acción intersectorial pero acotada a temas específicos como alimentación saludable, control de tabaco y alcohol, reducción de accidentes de tránsito, violencia, entre otros. En tanto que la segunda definición parte de una visión holística basada en los determinantes sociales estructurales de la salud, y abre un gran espectro de ámbitos de acción sectoriales y políticas sociales y económicas.

Por otra parte, se ha visto que cuando las políticas públicas en salud se orientan hacia una mayor equidad en el acceso a los servicios de salud curativos, esta

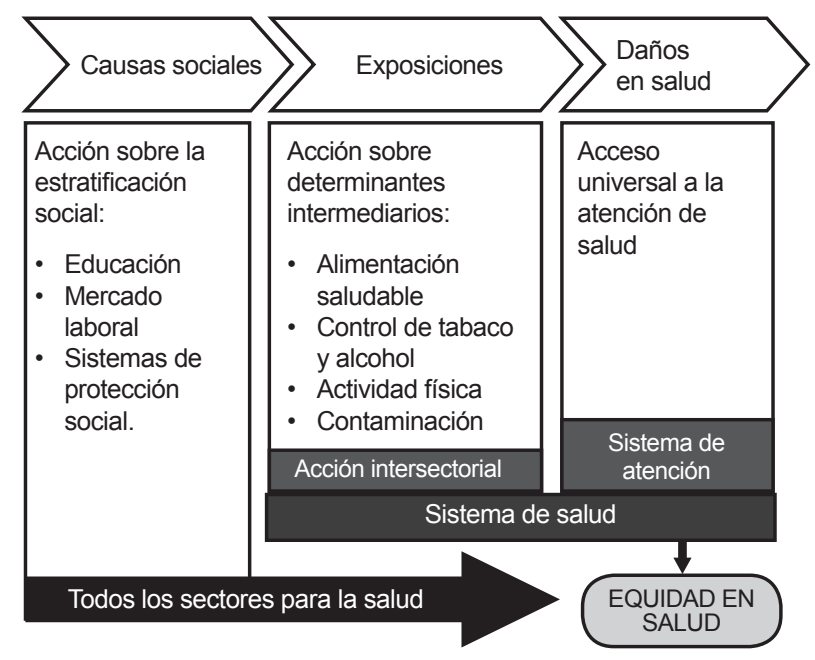

Figura 1. Concepto de salud y ámbito de políticas Fuente: elaboración propia

suele ser sectorial y se enfoca en el acceso universal a los servicios de atención médica, con especial atención a los grupos sociales desaventajados, excluidos de la seguridad social, como los pobres y los trabajadores informales. También ha existido preocupación por mejorar los servicios prestados por la seguridad social.

La reciente ola de reformas en la región, iniciadas en la primera década del presente siglo, muestra una mayor vocación universal y equitativa que las reformas experimentadas durante las dos últimas décadas del siglo XX. En general, las reformas recientes apuntan a la cobertura efectiva de las necesidades sanitarias, con niveles mínimos de servicios de salud, abarcando la atención primaria y, con cada vez más frecuencia, prestaciones más integrales y de mayor complejidad. El espacio de la equidad de acceso depende de la amplitud del conjunto de servicios a universalizar: un paquete básico, servicios comprensivos, o todos los servicios. Además, los resultados en equidad dependen del grado de preocupación para las necesidades diferenciales y las barreras específicas que enfrentan las poblaciones según sus circunstancias sociales.

Si bien las reformas han apuntado al establecimiento y universalización de niveles mínimos de cobertura (con la esperanza de que se expandan en el tiempo), se observan diferentes estrategias en las políticas públicas seguidas por los países. El plan AUGE en Chile, el Seguro Popular en México y la reforma de la salud en Colombia, todos ellos descritos en la Tabla 1, son algunos ejemplos ${ }^{(7-9)}$. Por su parte, Brasil ha optado por una estrategia que no descansa en mínimos garantizables, sino que buscó la universalización en el acceso a través de la creación de un Sistema Único de Salud (SUS), en el cual, en principio, 
Tabla 1. Ejemplos de reformas para universalizar la atención de salud y mejorar la equidad

\begin{tabular}{|c|c|c|}
\hline País & Reforma & Descripción \\
\hline Brasil & $\begin{array}{l}\text { Sistema } \\
\text { Único de } \\
\text { Salud }\end{array}$ & $\begin{array}{l}\text { La Constitución de } 1988 \text { transformó el antiguo sistema de seguridad social en un derecho universal e integral } \\
\text { a la salud para toda la población. Los ejes estratégicos son la descentralización, universalidad, equidad e } \\
\text { integralidad en la atención, con control social participativo. El Sistema Único de Salud (SUS) es gratuito para toda } \\
\text { la población y se financia con impuestos generales y contribuciones. La provisión es descentralizada a través } \\
\text { de establecimientos propios y servicios contratados con el sector privado. El subsector privado, utilizado por los } \\
\text { estratos de ingreso medio y alto, es voluntario y complementario. No obstante, la población con seguro privado } \\
\text { también utiliza los servicios del SUS, especialmente para atención compleja y costosa. }\end{array}$ \\
\hline Chile & AUGE & $\begin{array}{l}\text { Bajo la Constitución de 1981, los chilenos tienen derecho a elegir entre el sistema público o privado. La } \\
\text { segmentación y el carácter dual del sistema mixto de salud concentra en el seguro social público, el Fondo } \\
\text { Nacional de Salud (FONASA), a los individuos de menor ingreso, mayor edad y peor estado de salud. Para } \\
\text { contrarrestar las inequidades inherentes al sistema dual, la reforma de salud chilena de } 2000-2005 \text { estableció el } \\
\text { sistema AUGE de atención garantizada para prioridades sanitarias, universal para los afiliados del sistema público } \\
\text { y privado. Según el presidente Ricardo Lagos: "Para resguardar el derecho a la salud no basta su declaración, } \\
\text { sino que es necesario establecer garantías explícitas de acceso, oportunidad, calidad y protección financiera". } \\
\text { Cumplir las garantías requirió incrementar el financiamiento público y establecer una nueva institucionalidad de } \\
\text { autoridad sanitaria. }\end{array}$ \\
\hline México & $\begin{array}{l}\text { Seguro } \\
\text { Popular }\end{array}$ & $\begin{array}{l}\text { La constitución de } 1983 \text { consagró el derecho a la salud a toda la población que se concretó con la reforma de } \\
\text { salud de } 2003 \text {, que estableció el Sistema de Protección Social en Salud. El Seguro Popular ofrece cobertura } \\
\text { universal a un paquete integral de servicios de salud con protección financiera para los más pobres. Hay cinco } \\
\text { seguros sociales que legalmente cubren a diversos segmentos de la población en el sector formal, la mayoría } \\
\text { de ingreso medio. Con el norte de garantizar el ejercicio efectivo del derecho a la salud, la visión de la reforma } \\
\text { fue de reorganizar el sistema de salud por funciones, mejorar la equidad y la eficiencia y alcanzar la cobertura } \\
\text { universal. Se fortaleció la función de rectoría del Ministerio de Salud, introdujo un nuevo esquema de financia- } \\
\text { miento público y consolidó la provisión con prestadores acreditados, públicos y privados. }\end{array}$ \\
\hline Colombia & Ley 100 & $\begin{array}{l}\text { La constitución de } 1991 \text { y la ley de } 1993 \text { ordenaron la universalidad de aseguramiento bajo el Sistema General } \\
\text { de Seguridad Social en Salud (SGSSS) con dos regímenes: el contributivo (RC) para trabajadores y pensiona- } \\
\text { dos, y el subsidiado (RS) que incluye a las personas sin capacidad de pago. Además existen regímenes espe- } \\
\text { ciales (RE) para las fuerzas militares, la policía, y otros grupos. La afiliación al SGSSS es obligatoria, mediada } \\
\text { por entidades promotoras de salud (EPS), públicas o privadas, que ofrecen planes obligatorios de servicios. Las } \\
\text { EPS transfieren los fondos recaudados por cotizaciones al Fondo de Solidaridad y Garantía, el cual paga a las } \\
\text { EPS una unidad per cápita ajustado por riesgo, de acuerdo con el número de afiliados. Tanto el per cápita como } \\
\text { el plan obligatorio son establecidos por el gobierno. }\end{array}$ \\
\hline
\end{tabular}

Modificado de Mesa-Lago ${ }^{(7)}$, Frenz et al. ${ }^{(8)}$ y Knaul et al.(9)

no hay restricciones a los tipos de servicios de salud a los que se puede tener acceso de manera universal. No obstante, la realidad de la restricción de recursos introduce racionamiento, al menos en tiempo de espera, y hay espacios para seguros suplementarios y servicios privados de salud ${ }^{(10,11)}$. Una de las lecciones de la última generación de reformas en la región tiene que ver con la ambigüedad en la definición del espacio de equidad. Al respecto, nos debemos preguntar si este significa la provisión universal de un paquete básico de servicios, la atención integral, o abarca acción sobre el conjunto de determinantes sociales de la salud ${ }^{(6)}$.

\section{LAS POLÍTICAS PÚBLICAS PARA LA PROMOCIÓN DE LA SALUD: ACCIONES DE SALUD PÚBLICA CON OTROS SECTORES.}

Por lo general, las reformas sanitarias tienden a ser más que reformas del sistema de atención individual de la salud, en reconocimiento a la importancia de las acciones de salud pública para la salud poblacional. Es decir, las reformas buscan transformar el sistema en un sistema de salud y no solamente un sistema de atención de la salud. Por ejemplo, aunque la reforma chilena se centró en fijar niveles garantizados de atención, en su concepción más general, un cambio importante fue la nueva institucionalidad de autoridad sanitaria, que buscaba fortalecer las acciones de salud pública, concebidas desde el enfoque de equidad y determinantes sociales.

Se reconoce el impacto de las acciones de otros sectores para proteger la salud y promover un país más saludable. Las políticas de control de tabaco, la regulación del alcohol, la seguridad de tránsito, la descontaminación ambiental, la seguridad alimentaria y la nutrición pública, han sido abordadas con este enfoque en Europa y -cada vez más- en América Latina ${ }^{(12)}$. El ejemplo paradigmático 
de política pública intersectorial lo demostró Finlandia cuando logró disminuir la mortalidad cardiovascular mediante la eliminación de los subsidios de alimentos altos en grasas, la promoción del cultivo de frutas y verduras, y la inversión en espacios públicos. Con ello generó un entorno sano para la población, logrando mejor salud para todos además de beneficios económicos.

Este trabajo intersectorial comenzó con un abordaje de problemas específicos, pero en la medida que se instala el enfoque de determinantes sociales y equidad se ha ido derivando hacia un trabajo más sistemático de transformación legal y estructural, en una perspectiva conocida como "salud en todas las políticas" (13).

\section{LA POLÍTICA PÚBLICA Y LOS DETERMINANTES SOCIALES ESTRUCTURALES: LA POLÍTICA SOCIAL INTEGRAL}

La salud es importante, ya sea porque es un bien primario, esencial para el funcionamiento humano y central para aprovechar oportunidades, o por ser inherente a la vida que queremos. La noción de justicia tiene que ver con la igualdad en el estado de la salud, lo que se reconoce cuando se afirma que la salud es un derecho ${ }^{(14)}$. En consecuencia, para alcanzar la equidad en salud, el objetivo de la política pública es necesariamente amplio: lograr el nivel máximo alcanzable de salud poblacional sin diferencias sociales. Esto obliga a actuar sobre las raíces socioeconómicas de las diferencias en salud, los llamados determinantes sociales estructurales de las inequidades en la salud, lo que abarcaría una amplitud de sectores y políticas públicas, mucho más allá de la provisión de servicios básicos de atención en salud y la promoción intersectorial. En el fondo, el conjunto de políticas económicas y sociales que impactan en la estratificación social y las condiciones de vida y trabajo de la población son fundamentales para lograr el objetivo de equidad sanitaria. Además, significa actuar sobre el gradiente social, a través de políticas universales pero proporcionadas, que logren que los beneficios se incrementen a lo largo del gradiente ${ }^{(15)}$.

Los objetivos de las políticas sociales son amplios y diversos. Crecientemente se plasman en la región políticas sociales integradas que van más allá de objetivos sectoriales y que buscan contribuir a la equidad social y en salud. Sin embargo, en alrededor de veinte países de América Latina se han puesto en marcha programas de transferencia condicionada de recursos monetarios y no-monetarios, más un conjunto de servicios sociales para familias que viven en pobreza, todos relacionados con el cumplimiento de objetivos sociales en salud, educación, y trabajo ${ }^{(16)}$. Cabe preguntarse si estos programas representan la universalización de las garantías sociales con beneficios proporcionales a las necesidades de los grupos vulnerables, en un enfoque de universalismo proporcionado, o solamente son políticas focalizadas ${ }^{(15)}$.

Entre estos se puede mencionar el programa de protección social Chile Solidario, que recluta y apoya a las familias pobres y marginales con la entrega de garantías sociales en diversas dimensiones de vida, tales como educación, trabajo, vivienda, habitabilidad, e identidad, siendo la salud una de ellas. Aún más profundo cuando se conoce su declaración de equidad: Eliminar las diferencias sociales en el desarrollo de los niños y niñas en Chile. El programa Chile Crece Contigo constituye un sistema integral de protección a la infancia, cuya puerta de entrada es el primer control prenatal en el centro de salud familiar (17). Sin embargo, a pesar de su anunciado épico se debe reconocer que, nuevamente, es un ejemplo de derechos sociales garantizados con estándares mínimos, un nivel universal.

Otro ejemplo de una política pública que persigue una aproximación integral de "salud en todas las políticas" es la agenda de equidad y determinantes sociales impulsada por la Subsecretaria de Salud Pública de Chile entre 2008 y 2010 . Esta iniciativa intentó poner en marcha acciones concretas para adoptar las recomendaciones de la Comisión sobre Determinantes Sociales de la Salud de la OMS, estableciendo trece pasos para la equidad en salud (Tabla 2), que se traducirían en políticas de salud y de otros sectores orientadas a reducir inequidades en salud ${ }^{(18)}$.

\section{FORTALECIMIENTO DEL ESTADO: UNA AGENDA INSTITUCIONAL}

Uno de los principales desafíos de las políticas públicas en la región se refiere a la capacidad que tienen nuestros Estados de financiar, diseñar, implementar, evaluar, y monitorear las políticas. En este sentido, avanzar hacia una mayor equidad en salud requiere un doble desafío. No solo es necesario diseñar e implementar políticas sectoriales o intersectoriales, sino que también se requiere fortalecer la capacidad institucional de los Estados.

Un ejemplo de los desafíos institucionales para las políticas públicas que involucran avanzar hacia la equidad en salud se refleja en la agenda de equidad y determinantes sociales de Chile (Tabla 2). Los pasos conducen al fortalecimiento de las capacidades del sector salud y otros sectores económicos y sociales a través del desarrollo de conocimientos, espacios de debate, formación, planificación, monitoreo y evaluación, e instancias de coordinación y participación, tanto a nivel local, nacional como internacional ${ }^{(18)}$. 
Tabla 2. Los trece pasos de la agenda de equidad de la Subsecretaría de Salud Pública de Chile

\begin{tabular}{|c|c|}
\hline Pasos & Objetivo \\
\hline $\begin{array}{l}\text { Paso 1: desarrollo temprano saludable y seguro: Los niños y niñas tienen derecho } \\
\text { a iguales oportunidades de desarrollo. } \\
\text { Paso 2: condiciones de empleo y trabajo que contribuyen a la salud y una vida } \\
\text { laboral inclusiva. }\end{array}$ & $\begin{array}{l}\text { Reducir las inequidades sociales que } \\
\text { contribuyen a las inequidades en salud }\end{array}$ \\
\hline $\begin{array}{l}\text { Paso 3: iniciar un proceso de rediseño de los programas de salud desde la } \\
\text { perspectiva de equidad y determinantes sociales. } \\
\text { Pasó 4: fortalecer las estrategias poblacionales que promueven cambios de } \\
\text { exposición y conductas de riesgo no saludables, tales como: alimentación, } \\
\text { tabaquismo, alcoholismo y sedentarismo. }\end{array}$ & $\begin{array}{l}\text { Reducir las inequidades sociales que } \\
\text { contribuyen a un menor acceso a la salud y los } \\
\text { servicios de salud. }\end{array}$ \\
\hline $\begin{array}{l}\text { Pasó 5: iniciativas conjuntas para fortalecer programas regionales de "inclusión } \\
\text { social" hacia grupos de trabajadores de mayor desprotección: pescadores } \\
\text { artesanales, pirquineros, recolectores y temporeros entre otros. } \\
\text { Paso 6: fortalecer acciones integradas en zonas geográficas priorizadas. }\end{array}$ & $\begin{array}{l}\text { Implementar acciones de "inclusión social' } \\
\text { sobre grupos y zonas excluidos. }\end{array}$ \\
\hline $\begin{array}{l}\text { Paso 7: elaborar e implementar el año } 2009 \text {, un programa de capacitación } \\
\text { "Fundamentos para la integración de equidad y determinantes sociales" en el } \\
\text { diseño e implementación de políticas públicas y programas de salud. }\end{array}$ & $\begin{array}{l}\text { Desarrollo de conocimientos y espacios de } \\
\text { debate y formación en equidad y determinantes } \\
\text { sociales. }\end{array}$ \\
\hline $\begin{array}{l}\text { Paso 8: completar diagnósticos territoriales a nivel regional y comunal que } \\
\text { incorporen un análisis de equidad y determinantes sociales de la salud. } \\
\text { Paso 9: implementar un sistema de monitoreo y evaluación de intervenciones } \\
\text { efectivas en equidad en salud implementadas en Chile a nivel local y nacional. } \\
\text { Paso 10: implementar mecanismos y espacios de participación para el monitoreo y } \\
\text { evaluación, "control social" sobre el compromiso de abordar las inequidades en salud. }\end{array}$ & $\begin{array}{l}\text { Generar herramientas de planificación } \\
\text { monitoreo y evaluación para el proceso de } \\
\text { instalación del enfoque de DSS y equidad en e } \\
\text { trabajo del Ministerio de Salud. }\end{array}$ \\
\hline $\begin{array}{l}\text { Paso 11: generar y fortalecer espacios de coordinación, intersectorialidad y de } \\
\text { evaluación conjunta con otras sectores; salud en todas las políticas. }\end{array}$ & $\begin{array}{l}\text { Establecer instancias que permitan la } \\
\text { coordinación e integración entre distintos } \\
\text { sectores para el desarrollo de acciones de } \\
\text { abordaje de las inequidades en salud. }\end{array}$ \\
\hline $\begin{array}{l}\text { Paso 12: desarrollo de foros de discusión y diálogo a nivel provincial, regional y } \\
\text { nacional. } \\
\text { Paso 13: establecer acciones de colaboración e intercambio con países de la } \\
\text { región, con organizaciones internacionales, tales como OMS / OPS y otros países } \\
\text { fuera de la región. }\end{array}$ & $\begin{array}{l}\text { Abogar por la agenda de equidad y } \\
\text { determinantes de la salud en el país y la región } \\
\text { de las Américas. }\end{array}$ \\
\hline
\end{tabular}

Modificado de Vega J. Steps towards towards the health equity agenda in Chile (18)

Cabe resaltar que la construcción conjunta de la salud entre el personal de salud, los agentes comunitarios y las familias, a través de la negociación y el empoderamiento en el ejercicio de los derechos, solo se logra con la participación activa de la comunidad y de las familias e individuos. Perú lo ha impulsado con las Comunidades Locales de Administración de Salud (CLAS), en el marco general de la cogestión y participación ciudadana para los establecimientos de salud del primer nivel de atención del Ministerio de Salud, y de los gobiernos regionales y locales. Ellas contribuyen a ampliar la cobertura, mejorar la calidad y el acceso equitativo a los servicios de salud, generar mejores condiciones sanitarias y garantizar el ejercicio del derecho a la salud ${ }^{(19)}$.

Por último, un factor central en el fortalecimiento institucional de los Estados es la capacidad que estos tengan para financiar las políticas públicas en salud ya sean sectoriales o intersectoriales. Esto cobra mayor relevancia en la medida que el componente no contributivo de la protección social en salud sea un elemento central de las políticas de universalización del derecho a la salud, reflejadas en mejores condiciones de salud y calidad de vida. Así, la provisión de bienes públicos que mejoren las condiciones de salud de la población debe ser un eje central de las políticas públicas. Lo anterior requiere que los países de la región hagan esfuerzos importantes en materia fiscal para poder movilizar los recursos requeridos ${ }^{(20)}$.

\section{CONCLUSIONES}

Las estrategias para universalizar la cobertura del derecho a la salud y los derechos sociales en la región han llevado a desarrollar políticas públicas dependiendo de la definición y operacionalización de los criterios de equidad. En este sentido, la región ha privilegiado las políticas públicas sectoriales, concentrándose en reformas al sector salud, y solo recientemente hay esbozos de políticas que tiendan a una mayor integración intersectorial. 
En general, los enfoques de paquetes mínimos ponen un nivel de igualdad, pero dejan un techo de diferencia. Estos enfoques no apuntan a la igualdad porque mantienen la segregación y permiten diferentes accesos (derechos) a diversos grupos poblacionales y distan mucho de sistemas verdaderamente universales y equitativos. El pragmatismo impuesto por la escasez de recursos no debe hacernos olvidar hacia donde queremos llegar: la igualdad de derechos de todos ciudadanos. Si la equidad en salud es nuestro norte, establecer garantías mínimas no puede ser la respuesta final sino un paso en el camino hacia la igualdad. Asimismo, avanzar hacia una cobertura universal de los derechos en salud requiere fortalecer las capacidades institucionales de los gobiernos tanto en sus diseños e implementación como en su capacidad de financiamiento.

Contribuciones de autoría: PF y DT han participado de la concepción del artículo, de la recolección e interpretación de datos, de la redacción y aprobación de la versión final.

Fuentes de financiamiento: autofinanciado.

Conflictos de interés: los autores declaran no tener conflictos de interés.

\section{REFERENCIAS BIBLIOGRÁFICAS}

1. Comisión Económica para América Latina y el Caribe (CEPAL). La hora de la igualdad: brechas por cerrar, caminos por abrir. Santiago: Comisión Económica para América Latina y el Caribe; 2010.

2. Programa de las Naciones Unidas para el Desarrollo (PNUD). Informe sobre Desarrollo Humano 2013. El ascenso del Sur: Progreso humano en un mundo diverso. Nueva York: Programa de Naciones Unidas para el Desarrollo; 2013.

3. Lang T, Kaminski M, Leclerc A. Report of the WHO commission on social determinants of health: a French perspective. Eur J Public Health. 2009 Apr;19(2):133-5. doi: 10.1093/ eurpub/ckp025.

4. Etienne CF. Líder de la salud de las Américas: desarrollo sostenible y equidad. PAHO/AMRO Regional Director confirmation speech [Internet]. Washington, DC: World Health Organization; 2013 [citado el 15 de mayo de 2013]. Disponible en: http://www.paho.org/hq/index. php?option $=$ com_content\&view $=$ cate gory\&layout $=\mathrm{blog} \& \mathrm{id}=651 \&$ Itemid $=$ 291\&lang=es

5. Whitehead M. The concepts and principles of equity and health. Int J Health Serv. 1992;22(3):429-45.

6. O’Connell T, Rasanathan K, Chopra M. What does universal health coverage mean? Lancet. 2013;6736(13):13-5.

7. Mesa-Lago C. Las reformas de salud en América Latina y el Caribe: su impacto en los principios de la seguridad social [Internet]. Santiago: CEPAL; 2005 [citado el 15 de mayo de 2013]. Disponible en: http://www. eclac.cl/cgi-bin/getProd.asp? xml=/
publicaciones/xml/8/24058/P24058.

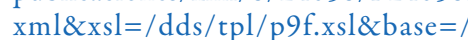
$\mathrm{dds} / \mathrm{tpl} /$ top-bottom.xsl

8. Frenz P, Delgado I, Villanueva L, Kaufman JS, Muñoz F, Navarrete MS. Seguimiento de cobertura sanitaria universal en Chile entre 2000 y 2011 usando las Encuestas CASEN. Rev Med Chile. 2013;141:1095-106.

9. Knaul FM, González-Pier E, Gómez-Dantés O, García-Junco D, Arreola-Ornelas H, Barraza-Lloréns $\mathrm{M}$, et al. The quest for universal health coverage: achieving social protection for all in Mexico. Lancet. 2012;380(9849):1259-79.

10. Tolentino Silva M. Sistema único de salud: la experiencia brasileña en la universalización del acceso a la salud. Rev Peru Med Exp Salud Publica. 2009;26(2):251-7.

11. Paim J, Travassos C, Almeida C, Bahia L, Macinko J. The Brazilian health system: history, advances, and challenges. Lancet. 2011;377(9779):1778-97.

12. Mackenbach JP, Karanikolos M, McKee M. The unequal health of Europeans: successes and failures of policies. Lancet. 2013;381(9872):1125-34.

13. Melkas T. Health in all policies as a priority in Finnish health policy: a case study on national health policy development. Scand J Public Health. 2013;41(11 Suppl):3-28.

14. Sen A. ¿Por qué la equidad en salud? Rev Panam Salud Publica. 2002;11(56):302-9.

15. Benach J, Malmusi D, Yasui Y, Martínez JM. A new typology of policies to tackle health inequalities and scenarios of impact based on Rose's population approach. J
Epidemiol Community Health. 2013;67(3):286-91.

16. Cecchini S, Madariaga A. Conditional Cash Transfer Programmes. The recent experience in Latin America and the Caribbean [Internet]. ECLAC, editor. Santiago; 2011 [citado el 15 de mayo de 2013]. Disponible en: http://www. eclac.org/cgi-bin/getProd.asp?xml=/ publicaciones/xml/6/45096/P45096. $\mathrm{xml} \&$ base $=/ \mathrm{mujer} / \mathrm{tpl} /$ top-bottomestadistica.xslt

17. Frenz P. Innovative practices for intersectoral action on health: a case study of four programs for social equity. Santiago: WHO Commission on Social Determinants of Health; 2007.

18. Vega J. Steps towards towards the health equity agenda in Chile [Internet]. Río de Janeiro: World Health Organization; 2011 [citado el 15 de mayo de 2013]. Disponible en: http://www.who. int/sdhconference/resources/draft background_paper25_chile.pdf

19. Ley que establece la Co-gestión y Participación Ciudadana para el Primer Nivel de Atención en los establecimientos de salud del Ministerio de Salud y de las Regiones. Ley No 29124. Perú, 12 de octubre de 2007.

20. Titelman D. Acceso y cobertura de los sistemas de salud en América Latina: una agenda pendiente. Reformas para la Cohesión Social en América Latina. Santiago: CEPAL/CIDOB; 2008.

Correspondencia: Patricia Frenz

Dirección: Escuela de Salud Pública,

Facultad de Medicina, Universidad de

Chile. Independencia 939, Independencia.

Santiago, Chile

Teléfono: 56-9-98206994

Correoelectrónico:pfrenz@med.uchile.cl 